\title{
Disorder and non-conservation in a driven diffusive system
}

\author{
M. R. Evans ${ }^{1}$, T. Hanney ${ }^{1}$ and Y. Kafri ${ }^{2}$ \\ ${ }^{1}$ School of Physics, University of Edinburgh, Mayfield \\ Road, Edinburgh, EH9 3JZ, United Kingdom \\ ${ }^{2}$ Department of Physics, Harvard University, Cambridge, MA 02138
}

\begin{abstract}
We consider a disordered asymmetric exclusion process in which randomly chosen sites do not conserve particle number. The model is motivated by features of many interacting molecular motors such as RNA polymerases. We solve the steady state exactly in the two limits of infinite and vanishing non-conserving rates. The first limit is used as an approximation to large but finite rates and allows the study of Griffiths singularities in a nonequilibrium steady state despite the absence of any transition in the pure model. The disorder is also shown to induce a stretched exponential decay of system density with stretching exponent $\phi=2 / 5$.
\end{abstract}




\section{INTRODUCTION}

Driven diffusive systems serve as simple models for collective phenomena ranging from traffic flow to molecular motors. Moreover, they provide tractable examples of systems far from thermal equilibrium. Studies of one-dimensional driven diffusive systems have shown that many interesting phenomena, which are typically not observed in one dimensional systems in thermal equilibrium, exist. Prominent examples are boundary induced phase transitions and spontaneous symmetry breaking, for reviews see e.g. [1, 2, 3].

Most studies have considered systems in which the dynamics are the same everywhere in the system or systems where the dynamics are modified only at the boundaries. However, when trying to relate these systems to many interacting molecular motors, the effects of nonconservation and disorder (i.e. spatial heterogeneity in the dynamics) can not be ignored in many cases.

Indeed there have been some studies on the effects of disorder on driven diffusive systems. For example, the effect of assigning a disordered quenched rate to each particle was studied in [4, 5, 6, 7, 8, 9] on a ring geometry. Exact solutions show that at high enough densities a macroscopic number of particles jam behind the slowest particle in the system. The phase transition between the jammed and non-jammed phase is similar to a Bose-Einstein condensation. Work has also been done on an asymmetric exclusion process on a ring where the quenched hopping rates between neighboring sites are drawn at random [8, 9, 10]. For molecular motors moving along a disordered substrate this seems to be the relevant scenario [11, 12]. It was argued, based on numerics and mean-field solutions, that at high densities the system phase separates into a region of high density coexisting with a low density region. Finally, the combined effect of random hopping rates and open boundary conditions was considered in [13, 14]. In [13] it was argued using numerics that the location of phase transition lines may be sample dependent. In [14] mean-field arguments and numerics indicate the existence of shifts in phase boundaries which, by analogy with equilibrium systems, are expected to be accompanied by emergent Griffiths regions. A review of the effects of disorder in exclusion models has been given in [15].

In this paper we consider another type of disorder. We study an asymmetric exclusion process (ASEP) where non-conserving sites are chosen at random along the lattice. At these sites particles may attach and detach with specified rates which may also be drawn 
at random. Thus there are two components to the disorder. A feature of this disorder is that it allows a detailed account of the way in which Griffiths singularities can arise in nonequilibrium steady states. In equilibrium the mechanism leading to Griffiths singularities is well understood: the disorder, e.g. dilution, breaks the system into pure regions and large pure regions may give rise to the exponentially suppressed Griffiths singularities. In the present the case, in the limit of high attachment and detachment rates, the non-conserving sites break the system into driven conserving domains.

Non-conservation of particles in driven systems without disorder has previously been considered in the context of molecular motors. The idea is that molecular motors move in a preferred direction along a filament and are able to attach and detach from the filament. In the works so far all sites are non-conserving [16, 17, 18, 19]. The motivation for the model we study here comes from the fact that some molecular motors only attach and detach at certain sites.

More specifically, we give a very simplified description of many interacting RNA polymerase (RNAp) motors acting on a prokaryotic DNA in vitro. Prokaryotic RNA polymerase can initiate without regulatory proteins. Namely, RNAp left in a solution with DNA can produce RNA even if the specific protein which regulates its action (for example, by enhancing or reducing the initiation rates) is present. They can enter and leave the DNA in order to transcribe RNA molecules at specific sites, referred to as promotor and termination sites respectively. In the language of the lattice model we consider a binding of a RNAp to a promotor corresponds to a particle entering the system. The unbinding at the termination site corresponds to a particle leaving the system. In the absence of regulatory proteins the rate of entering the DNA depends on the details of the promotor sites. In such systems the RNA polymerase do not usually move from one gene to another. In the lattice model this would correspond to particles not moving from one stretch of conserving sites to a neighboring one i.e. the limit in which the detachment rates are large at the non-conserving sites. We comment that in principle RNA polymerase may move in different directions along the DNA when transcribing different messenger RNAs, corresponding to particles moving in different directions along different conserving stretches. However, in the limit when the detachment rate is large this will not influence most of the results described in the paper. Of course, our assumption of randomly distributed lengths of genes (or conserving segments) is not expected to hold. However, the model provides a starting point for analyzing more realistic 
situations.

The paper is organised as follows. In Section [1] we define the model and discuss two limits which are exactly soluble. In Section [II we show that the disorder induces Griffiths like singularities as the rates for entering and leaving the lattice are changed. More significantly, it is shown in Section $1 \nabla$ that the presence of the non-conserving sites leads to anomalous relaxation of the system toward the steady state. Specifically, we argue that decay of measurable quantities decay as a function of time, $t$, as a stretched exponential $\exp \left(-c t^{\phi}\right)$, where $c$ is a non-universal constant and $\phi=2 / 5$. The results are verified numerically. We conclude in Section $\nabla$

\section{MODEL}

The model we consider is a disordered generalisation of the ASEP. The pure ASEP is defined on a one-dimensional lattice containing $L$ sites and with periodic boundaries. The lattice is occupied by particles subject to an exclusion interaction, which prohibits multiple occupancy of any site. These particles hop with rate one to the nearest neighbour site to the right, provided it is empty, and so the total particle number $N$ is conserved. We introduce non-conservation into this model by allowing, at certain sites (which we will call 'disorder' sites), processes which do not conserve the total particle number $N$. Hence each site $l$ $(l=1, \ldots, L)$ in the pure model remains a pure site with probability $p$, or becomes a disorder site with probability $(1-p)$. Now, at the disorder sites, labelled by $j=1, \ldots, P$, particles attach with rate $c_{j}$ or detach with rate $a_{j}$. In general, we wish to consider heterogeneous rates for the non-conserving processes.

To study the model we first consider limits which can be solved exactly. Later, using numerics, we argue that the results are generic. Exact solubility arises when the steady state densities at the disorder sites are determined solely by the attachment and detachment processes. In these cases, since the system is composed of conserving domains of the chain in contact with disorder sites at the boundaries of each domain, the steady state can be written as a product of boundary driven ASEPs in which the densities of the boundary reservoirs are given by the disorder site densities $\rho_{j}$.

Before turning to the disordered ASEP under consideration we recap some facts, which will be useful later, about the boundary driven ASEP. For the boundary driven ASEP, in 
which particles are injected at the left-hand boundary site with rate $\alpha$ (provided it is vacant) and removed from the right-hand boundary site with rate $\beta$, exact steady state weights for particle configurations can be obtained using a matrix product ansatz [20]. In this ansatz, particle configurations are represented as a product of matrices $X_{1} \cdots X_{L}$ where $X_{l}=D$ $(E)$ if site $l$ is occupied (vacant). The steady state weight of a configuration is given by $\left\langle\alpha\left|X_{1} \cdots X_{L}\right| \beta\right\rangle$ provided the matrices $D$ and $E$ and the vectors $\langle\alpha|$ and $|\beta\rangle$ satisfy the relations

$$
D E=D+E \equiv C, \quad \alpha\langle\alpha| E=\langle\alpha| \quad \text { and } \quad \beta D|\beta\rangle=|\beta\rangle .
$$

From these relations exact expressions for the normalisation $\left\langle\alpha\left|C^{L}\right| \beta\right\rangle$ can be derived which show that the model undergoes a second order phase transition: when both $\alpha$ and $\beta \geq 1 / 2$ the system is in a maximum current phase, otherwise it is in one of two low current phases. The phase transition between the low current phases is first order. The rates $\alpha$ and $1-\beta$ represent the densities of particles in reservoirs connected to the boundary sites.

Next, we use the known results for the boundary driven ASEP to study the disordered case. As stated above, there are limits where the steady state weight of the disordered model factorizes into a product over boundary driven ASEP weights. The two exactly soluble limits are:

- $c_{j}, a_{j} \rightarrow \infty$, with $c_{j} / a_{j}$ fixed.

In this limit, each disorder site $j$ acquires a density $\rho_{j}$ determined solely by $c_{j}$ and $a_{j}$ which obeys the equation of motion,

$$
\frac{\partial \rho_{j}}{\partial t}=c_{j}\left(1-\rho_{j}\right)+a_{j} \rho_{j}
$$

Therefore in the steady state,

$$
\rho_{j}=\frac{c_{j}}{c_{j}+a_{j}} .
$$

If we define $n_{j}$ to be the number of sites between disorder sites $j$ and $j+1$ (i.e. the length of the $j$-th conserving domain), then the normalisation $Z_{L}\left(\left\{n_{j}\right\}\right)$ (which is the sum over the steady state weights of all particle configurations on sites excluding the disorder sites), for a given configuration of the disorder sites $\left\{n_{j}\right\}=n_{1}, \ldots, n_{P}$, factorises into a product over normalisations for the boundary driven ASEP:

$$
Z_{L}\left(\left\{n_{j}\right\}\right)=\prod_{j=1}^{P}\left\langle\rho_{j}\left|C^{n_{j}}\right| 1-\rho_{j+1}\right\rangle
$$


and where $\rho_{P+1}=\rho_{1}$. This is the relevant limit for the model molecular motors discussed in the introduction.

- $c_{j}, a_{j} \rightarrow 0$, with $c_{j} / a_{j}$ fixed.

In this limit the time between each attachment/detachment event tends to infinity. Therefore, after each event the system reaches a homogeneous steady state of the pure ASEP with periodic boundaries. Thus the system density $\rho=N / L$, satisfies the equation of motion

$$
\frac{\partial \rho}{\partial t}=\left[\sum_{j=1}^{P} c_{j}\right](1-\rho)+\left[\sum_{j=1}^{P} a_{j}\right] \rho .
$$

Therefore in the steady state the system density is given by

$$
\rho=\frac{\sum_{j=1}^{P} c_{j}}{\sum_{j=1}^{P}\left(c_{j}+a_{j}\right)} .
$$

Because the steady state is homogeneous, all sites, including disorder sites, have the same steady state density $\rho$. Moreover, the steady factorises and there are no correlations between sites. Therefore, one can still write the normalisation in a form similar to (41):

$$
Z_{L}\left(\left\{n_{j}\right\}\right)=\prod_{j=1}^{P}\left\langle\rho\left|C^{n_{j}}\right| 1-\rho\right\rangle .
$$

This is because in this case $D$ and $E$ are given by the scalars $1 /(1-\rho)$ and $1 / \rho$.

In the following we will consider the first limit, $c_{j}, a_{j} \rightarrow \infty$. We use the factorised form (44) with $\rho_{j}$ given by (3) as an approximation for the case where $c_{j}, a_{j}$ are large but finite which is relevant for the model of molecular motors. This approximation has a mean-field character, in the sense that correlations are factorised about the disorder sites, however all correlations within conserving domains are retained.

\section{GRIFFITHS SINGULARITIES}

We can exploit known properties of the normalisation of the boundary driven ASEP to demonstrate the existence of Griffiths-type singularities in the disordered ASEP [21, 22]. As an illustrative example, we consider binary disorder at disorder sites, such that

$$
\rho_{j}=\frac{c_{j}}{a_{j}+c_{j}}=\left\{\begin{array}{l}
u \text { with probability } q, \\
v \text { with probability } 1-q .
\end{array}\right.
$$


This is the simplest choice of disorder for which Griffiths singularities occur. Generalizations to more complicated situations are straightforward.

Using (4) the steady state normalization satisfies

$$
\ln Z_{L}=\sum_{j=1}^{P} W_{n_{j}}\left(\rho_{j}, 1-\rho_{j+1}\right),
$$

where $W_{n}\left(\rho_{j}, 1-\rho_{j+1}\right)=\ln \left\langle\rho_{j}\left|C^{n_{j}}\right| 1-\rho_{j+1}\right\rangle$. In order to perform the disorder average, we write (8) in the form

$$
\begin{aligned}
& \ln Z_{L}=\sum_{n=0}^{\infty}\left[\nu_{u, 1-v}(n) W_{n}(u, 1-v)+\nu_{u, 1-u}(n) W_{n}(u, 1-u)\right. \\
& \left.+\nu_{v, 1-v}(n) W_{n}(v, 1-v)+\nu_{v, 1-u}(n) W_{n}(v, 1-u)\right]
\end{aligned}
$$

where $\nu_{\alpha, \beta}(n)$ is the number of conserving domains of size $n$ bounded by disorder sites at densities $\alpha$ and $1-\beta$.

We can average over the configurations of the $\nu_{\alpha, \beta}(n)$ by calculating the expectation values $\left\langle\nu_{\alpha, \beta}(n)\right\rangle$ in the thermodynamic limit (the angled brackets denote a disorder average). This is achieved by observing that $\lim _{L \rightarrow \infty} L^{-1}\left\langle\nu_{\alpha, \beta}(n)\right\rangle$ is just the probability that a site is part of an $n$-site conserving domain bounded by disorder sites with densities $\alpha$ and $1-\beta$, hence

$$
\begin{aligned}
\lim _{L \rightarrow \infty} L^{-1}\left\langle\ln Z_{L}\right\rangle=(1-p)^{2} \sum_{n=0}^{\infty} p^{n}\left\{q^{2} W_{n}(u, 1-u)+q(1-q)\left[W_{n}(u, 1-v)\right.\right. \\
\left.\left.+W_{n}(v, 1-u)\right]+(1-q)^{2} W_{n}(v, 1-v)\right\}
\end{aligned}
$$

The form of equation (10) is typical of systems which exhibit Griffiths singularities. In equilibrium, these singularities are usually inferred from the properties of the Yang-Lee zeros of the partition function - we can use the known properties of the Yang-Lee zeros of the the analogous quantity, the normalisation [23], to show how Griffiths singularities arise in the disordered nonequilibrium model: For fixed $u \geq 1 / 2$ say, in the complex $v$-plane and for $n$ arbitrarily large, the zeros of $\left\langle u\left|C^{n}\right| 1-v\right\rangle$ accumulate arbitrarily close to the point $v=1 / 2$ on the real axis. Therefore there exists a singularity in $W_{n}(u, 1-v)$ arbitrarily close to the point $v=1 / 2$ which is exponentially suppressed (by a factor $p^{n}$ ). Thus, such a Griffiths-type singularity follows whenever $u$ and $v$ are such that at least one of the $W_{n}(\alpha, \beta)$ in (10) lies on the phase boundary of the ASEP i.e. whenever $u$ and/or $v=1 / 2$.

One can go further and consider disorder in the $c_{j}$ 's and $a_{j}$ 's explicitly. For instance, if both $c_{j}$ and $a_{j}$ are drawn from binary distributions, then the densities at the disorder sites 
can assume one of four possible values, each with a different probability in general. However Griffiths singularities still arise whenever any one of these values for the density is $1 / 2$, as before.

It is also straightforward to use standard arguments from the study of dilute systems to show that the correlation length remains finite at the Griffiths singularity, as is the case in equilibrium systems. Note that Griffiths singularities emerge in this non-equilibrium system despite the absence of a transition in the pure model. This is in sharp contrast to equilibrium systems.

\section{DYNAMICS: STRETCHED EXPONENTIAL DECAY OF THE DENSITY TO ITS STEADY STATE VALUE}

In the pure boundary driven ASEP, whenever the system is in or at the boundary of the maximum current phase, the system density decays with time to its stationary value as an exponential with a decay constant that depends on system size $L$ as $L^{z}$, where $z=3 / 2$ is

the dynamic exponent 24, 25]. In the low current phase when the boundary injection and ejection rates are equal, a shock exists in the steady state, and the dynamic exponent $z=2$. Otherwise, in the low current phases the relaxation time is finite 24] and does not depend on $L$ Hence, in the disordered model, whenever contributions to the normalisation (10) are in the maximum current phase, the decay of the system density will be determined by the relaxation of these conserving domains.

For the following analysis, it is sufficient to consider disorder only in the location of the disorder sites: we consider homogeneous attachment and detachment rates i.e. $c_{j}=c$ and $a_{j}=a$. In the case where $c=a$, (3) gives $\rho=1 / 2$ at which point conserving domains are on the boundary of the maximum current phase; otherwise the conserving domains are in the low current phases. Thus only when $c=a$ do we expect the decay constant associated with a conserving domain to depend on its size [25].

Therefore for $c=a$, in a conserving domain of length $n$, we assume that the particle density $\rho_{n}(t)$ decays to its steady state value as

$$
\delta \rho_{n}(t) \equiv \rho_{n}(t)-1 / 2 \sim e^{-\Delta_{n} t}
$$

where $\Delta_{n}=\Delta_{0} n^{-3 / 2}$. In the disordered case, we need to sum over configurations of the 
disorder sites. This is achieved in the same way as in the previous section so, in the thermodynamic limit, the decay of the system density $\rho(t)$ becomes

$$
\delta \rho(t) \sim \sum_{n=0}^{\infty} p^{n} e^{-\Delta_{n} t},
$$

where we retain only the $n$-dependence, as is sufficient to determine the dominant contribution to the form of the relaxation. If we convert the sum into an integral and consider late times so that the integral can be evaluated at the saddle point, we obtain

$$
\delta \rho(t) \sim \exp \left(-c t^{\phi}\right)
$$

where $c$ is a constant and $\phi=(1+z)^{-1}=2 / 5$.

Equation (13) predicts the decay of the density up to some prefactor, power-law in $t$, with an exponent peculiar to the decay of the density. The stretching exponent $\phi$ should be universal however, in the sense that other correlation functions e.g. the current, should reach their stationary values with the same stretched exponential decay. This result should be valid for more general types of disorder whenever one has conserving domains in the maximal current phase.

In figures [1] and 2] we show the results of simulations. The simulations were run on systems of 10000 sites with periodic boundary conditions and averaged over 1000 histories of the dynamics, starting from an empty lattice and with the same realisation of disorder. The decay of the averaged system density $\delta \rho(t)$, is shown in Figure 1 for $a_{j}=c_{j}=10$, and in Figure 2 for $a_{j}=c_{j}=1$. In both cases the straight line $t^{2 / 5}$ is given for reference. Figure 11 shows very good agreement with the predicted stretched exponential decay, and even in figure 2, where $c_{j}$ and $a_{j}$ are not large, the agreement is still quite good. Thus it appears that our result for the stretching exponent holds for finite rates, although its derivation is only exact in the limit of infinite attachment and detachment rates.

\section{CONCLUSION}

In this work we have studied an ASEP with disorder sites where particles are not conserved. This may provide a basis for a more realistic model for interacting molecular motors

such as RNAp. We have used an approximation, exact in the limit of infinite non-conserving rates, the underlying assumption behind which is that the system factorises into conserving 


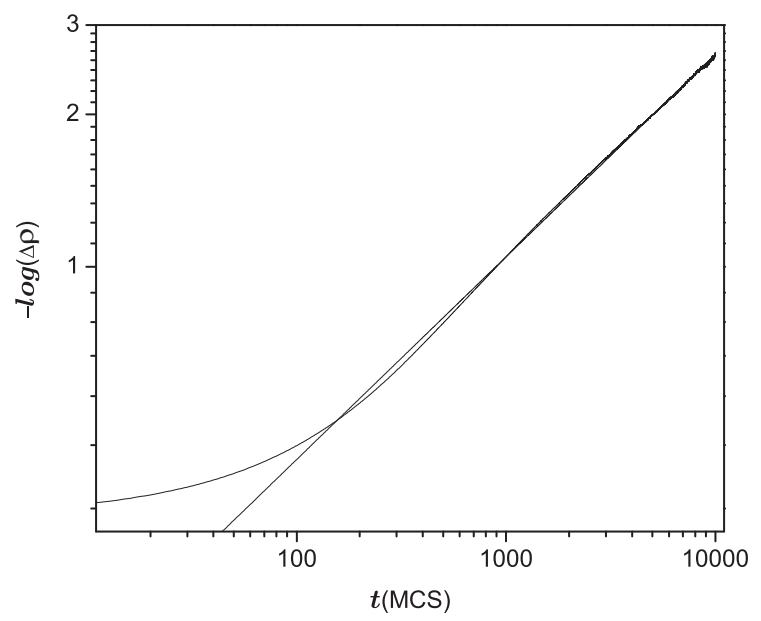

FIG. 1: Log-log plot of the decay of the density with time for $a_{j}=c_{j}=10$; the initial condition was an empty lattice.

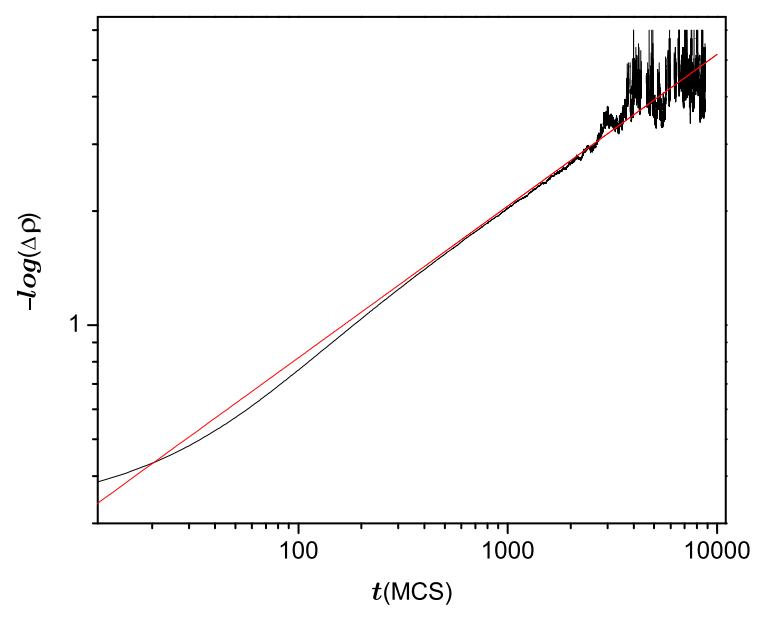

FIG. 2: Log-log plot of the decay of the density with time for $a_{j}=c_{j}=1$; the initial condition was an empty lattice.

domains. According to the ratios of the attachment and detachment rates these domains assume different phases of the ASEP with open boundaries.

Within this approximation we can explicitly identify Griffiths singularities. These arise when there are large conserving domains, on the boundary of the maximal current phase. An interesting feature is the prediction of a Griffiths singularity despite the absence of a transition in the pure system.

More generally one might ask under what conditions do Griffiths singularities arise in nonequilibrium steady states. In equilibrium systems Griffiths singularities are understood in terms of Yang-Lee zeros of the partition function. In nonequilibrium systems one does not have an energy function, nevertheless one can often identify a quantity that plays the role of a partition function, for example the normalisation (44), and recently there has been progress in understanding the zeros of such quantities [23].

When there is a spectrum of maximal-current conserving-domain sizes, we have demonstrated that correlation functions undergo a stretched exponential decay with a stretching exponent predicted to be $\phi=2 / 5$. Moreover simulations suggest this result holds for a wide range of attachment and detachment rates. A related stretched exponential decay has already been observed for the decay of autocorrelations in a bond diluted symmetric exclusion process on a ring [26] (in this case $z=2$ so $\phi=1 / 3$ ).

It would be instructive to develop further the approximation that the steady state fac- 
torises about the disorder sites. As we saw in Section II this approximation is exact in two limits, and we gave expressions for the densities at the disorder sites. It would be interesting to develop a scheme that interpolates between these two limits. Also of interest would be a better understanding of the correlations between the conserving domains which may exist away from the two exact limits and their effect on Griffiths singularities.

Acknowledgments The authors thank the Max Planck Institute for Complex Systems, Dresden, where this work was initiated, for hospitality. Work by Y.K was supported by the National Science Foundation through grant DMR-0229243 and grant DMR-0231631 and the Harvard Materials Research Laboratory via Grant DMR-0213805. MRE and TH were supported by EPSRC programme grant GR/S10377/01.

[1] D. Mukamel, in: Soft and Fragile Matter: Nonequilibrium dynamics, metastability and flow, eds. M.E. Cates and M.R. Evans (Bristol, Institute op Physics Publishing) (2000)

[2] M.R. Evans, Braz. J. Phys. 3042 (2000)

[3] G.M. Schütz, in: Phase Transitions and Critical Phenomena Vol. 19, eds. C. Domb and J. Lebowitz (London, Academic Press) (2001)

[4] J. Krug and P.A. Ferrari, J. Phys. A 29 L465 (1996)

[5] M.R. Evans, Europhys. Lett. 3613 (1996)

[6] M. Barma and K. Jain, Pramana-J. Phys. 58409 (2002)

[7] K. Jain and M. Barma, Phys. Rev. Lett. 91135701 (2003)

[8] G. Tripathy and M. Barma, Phys. Rev. E 581911 (1998)

[9] J. Krug, Braz. J. Phys. 3097 (2000)

[10] K.M. Kolwankar and A. Punnoose, Phys. Rev. E 612453 (2000)

[11] T. Harms and R. Lipowsky, Phys. Rev. Lett. 792895 (1997)

[12] Y. Kafri, D.K. Lubensky and D.R. Nelson, Biophys. J. in press.

[13] C. Enaud and B. Derrida, cond-mat/0402450

[14] R.J. Harris and R.B. Stinchcombe, cond-mat/0403062

[15] R.B. Stinchcombe, J. Phys.: Condens. Matter 141473 (2002)

[16] A. Parmeggiani, T. Franosch and E. Frey, Phys. Rev. Lett. 90086601 (2003)

[17] M.R. Evans, R. Juhász and L. Santen, Phys. Rev. E 68026117 (2003) 
[18] R. Lipowsky, S. Klumpp and T.M. Nieuwenhuizen, Phys. Rev. Lett. 87108101 (2001); S. Klumpp and R. Lipowsky, J. Stat. Phys. 113233 (2003)

[19] V. Popkov, A. Rakos, R. D. Willmann, A. B. Kolomeisky, G. M. Schütz, Phys. Rev. E 67 $066117(2003)$

[20] B. Derrida, M.R. Evans, V. Hakim and V. Pasquier, J. Phys. A 261493 (1993)

[21] R.B. Griffiths, Phys. Rev. Lett. 2317 (1969)

[22] M. Wortis, Phys. Rev. B 104665 (1974)

[23] R.A. Blythe and M.R. Evans, Braz. J. Phys. 33464 (2003); Phys. Rev. Lett. 89080601 (2002)

[24] U. Bilstein and B. Wehefritz, J. Phys. A 304925 (1997)

[25] Z. Nagy, C. Appert and L. Santen, J. Stat. Phys. 109623 (2002)

[26] M.D. Grynberg and R.B. Stinchcombe, Phys. Rev. E 61324 (2000) 